\title{
A Fast Matrix Element Method (FastME)
}

\section{Andre Sznajder ${ }^{* \dagger}$}

State University of Rio de Janeiro(UERJ) - Brazil

E-mail: andre.sznajderecern.ch

\section{Miqueias Melo de Almeida}

State University of Rio de Janeiro(UERJ) - Brazil

E-mail: miqueias.melo.de.almeida@cern.ch

We propose a Fast Matrix Element (FastME) method to determine event weights using a sample of simulated Monte Carlo events for a given process and its backgrounds, the events templates. Given a data event we search the signal and backgrounds template samples for the closest Monte Carlo events. This distance measure is defined in terms of the relevant final state particles in the event, taking into account the resolution of the measured observable quantities. The closest Monte Carlo event weight is used as an estimate for the data event weight, instead of evaluating the traditional Matrix Element method convolution integral between the process matrix element and the transfer functions. We show some preliminary results of the method performance.

XXVII International Symposium on Lepton Photon Interactions at High Energies 17-22 August 2015

Ljubljana, Slovenia

* Speaker.

${ }^{\dagger}$ A footnote may follow. 


\section{INTRODUCTION}

Matrix Element method is a multivariate data analysis method that explores in a maximal way the event information. Given a sample of experimental events and a set of theoretical models, the matrix element method is a procedure to select the most probable physics model associated to the production of these events. For each event, a weight is determined by the convolution of the squared matrix element of a given process and the a resolution function. The behavior of the squared matrix element require the use of specific parametrizations for the phase-space integration. Matrix Element is conceptually simple but implementation and numerical estimation is not straightforward. The Fast Matrix Element method(FastMe) is designed to estimate these weights using samples of simulated Monte Carlo events for a given process and its backgrounds, the events templates, without having to perform the integration of the matrix element.

\section{EVENT WEIGHTS}

Matrix Element method is based on the fact that the cross section for a process gives the probability that it occurs. The method attributes probability(weight) to events given a physics process hypothesis. The event weight is defined from the hadron level cross section according to

$$
P\left(x \mid \text { process }_{i}\right)=\frac{1}{\sigma_{i}} \frac{d \sigma_{i}}{d x}
$$

, where $x$ represents the event configuration(ex:particles 4-momenta).

The total cross section $\sigma_{i}$ ensures the normalization, so that $P\left(x \mid\right.$ process $\left._{i}\right)$ is the probability density for observing x ( ex: $P t, \eta$ ) given the process $\mathrm{i}$.

To relate the observable hadronic level cross $\operatorname{section}(\sigma)$ to the calculated parton level cross $\operatorname{section}(\hat{\sigma})$ the traditional Matrix Element method determine the event weight by integrating the following convolution of the partonic cross section

$$
P\left(x \mid \text { process }_{i}\right)=\frac{1}{\sigma_{i}} \int d y d q_{1} d q_{2} f\left(q_{1}\right) f\left(q_{2}\right) \frac{d \hat{\sigma}_{i}}{d y} W(x, y)
$$

, where the transfer function $W(x, y)$ gives the probability of an object of configuration $y$ being measured in the detector as $x$, This transfer function must be extracted from tunned MC or real data. The terms $f\left(q_{1}\right)$ and $f\left(q_{2}\right)$ are the hadrons PDFs and $\frac{d \hat{\sigma}_{i}}{d y}$ is the partonic level diferential cross section. This partonic cross section is proportional to the square of the matrix element $|M(y)|^{2}$ of the process.

\section{THE FAST MATRIX ELEMENT METHOD}

Instead of calculating weights through the integration of the convolution, the Fast Matrix Element (FastME) method uses samples of weighted Monte Carlo events to match with data events, based on phase space distance between theses events. It uses the already available weight of the closest MC event in the signal and background template samples as an estimate of the event weight according to the signal and backgrounds hypothesis. In other words, it uses samples of signal and 
backgrounds as lookup tables for the event weight. The choice of the distance definition is a key ingredient of the method and choose a $\chi^{2}$ type distance measure based on the following expression

$$
\text { Distance }=\sqrt{\sum_{i}\left[\frac{\left(P t_{i}^{\text {Data }}-P t_{i}^{M C}\right)^{2}}{\sigma_{P t}^{2}}+\frac{\left(\eta_{i}^{\text {Data }}-\eta_{i}^{M C}\right)^{2}}{\sigma_{\eta}^{2}}+\frac{\left(\phi_{i}^{\text {Data }}-\phi_{i}^{M C}\right)^{2}}{\sigma_{\phi}^{2}}\right]}
$$

, where the deviations for $P t, \eta$ and $\phi$ are normalized by the respective resolutions of theses quantities. One can also introduce some fudge factors to control the each deviation contribution.

We have performed some parton level studies using samples from Sherpa and Madgraph monte carlo event generators for the process $g g \rightarrow H \rightarrow W W \rightarrow 2 e 2 \mu$ and it's main background $q q \rightarrow W W \rightarrow 2 e 2 \mu$ which are presented in the distributions of figure 1 . On the left one finds the distribution of the discriminant for the closest events in each template, while on the right one has the distribution for the weights associated to these template events. Although we are not correcting for normalization on can see a sizable shape diference between signal and background.
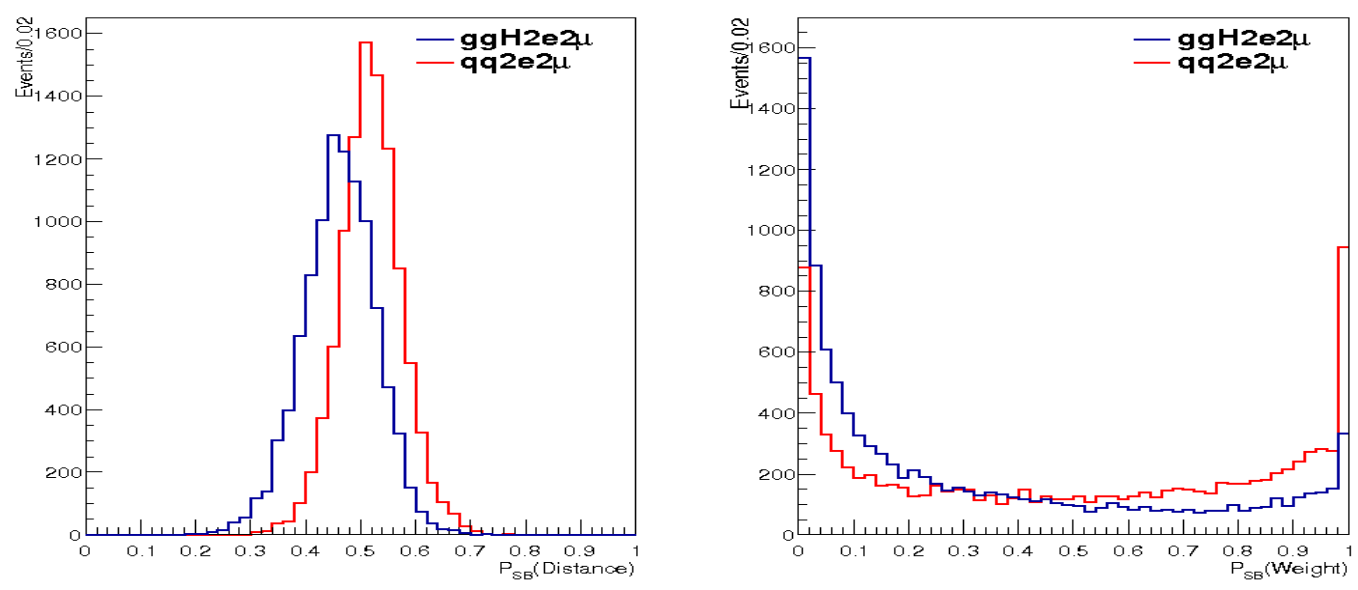

Figure 1: Discriminats based on distance (left) and based on weight (right) for the process Higgs decaying to 4 leptons and it's main background

In the figure 2 one finds the eficiency and purity for signal and background discrimination based on the above two discriminators.

\section{CONCLUSIONS}

Fast Matrix element based on the phase space distance from signal and background MC is a promising analysis tool for parameter estimation, hypothesis testing and signal versus background discrimination. It's based on very simple ideas and it can use any event generator capable of output weighted events for the signal and background event templates. 


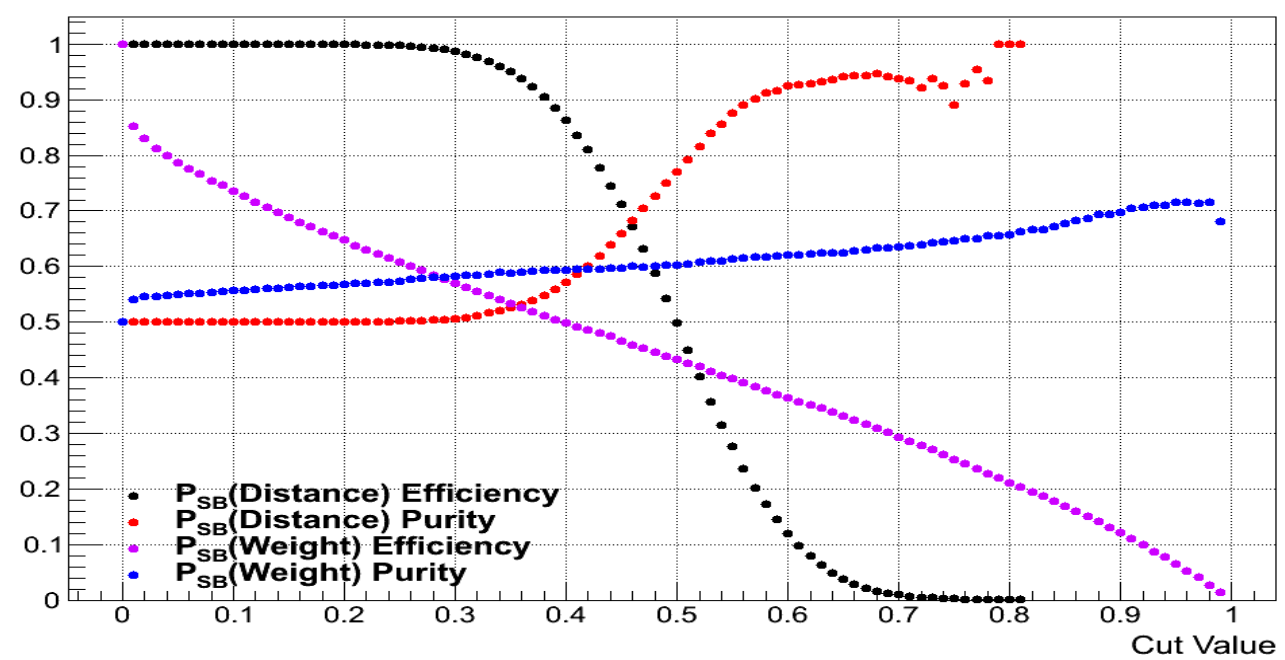

Figure 2: Efficiency and purity of the above discriminators

\section{BIBLIOGRAPHY}

1. P. Artoisenet et. all, Automation of the matrix element reweighting method ,arXiv:1007.3300v1

2. J. Alwall et. all , MadGraph 5: Going Beyond, JHEP1106(2011)128

3. A.Mertens, The automated Matrix-Element reweighting and its applications at the LHC, J.Phys.Conf.Ser. 523 (2014) 12028

4. T. Gleisberg et. all, Event generation with SHERPA 1.1, JHEP 0902 (2009) 007 